\title{
An Efficient Energy Saving Scheme Through Sorting Technique for Wireless Sensor Network
}

\author{
Hitesh Mohapatra ${ }^{1,3^{*}}$, Sourabh Debnath ${ }^{2}$, Amiya Kumar Rath ${ }^{3}$, Pravinkumar B Landge ${ }^{4}$, S. Gayen ${ }^{5}$, \\ R. Kumar ${ }^{6}$ \\ ${ }^{1}$ Department of Computer Science and Engineering, Koneru Lakshmaiah Education Foundation, \\ Vaddeswaram-522502, Guntur, Andhra Pradesh, India: hiteshmahapatra@gmail.com* \\ ${ }^{2}$ Department of CS\&E, National Institute of Technology, Rourkela, OD, India \\ ${ }^{3}$ Department of CS\&E, Veer Surendra Sai University of Technology, Burla, Sambalpur-768018, OD, India \\ ${ }^{4}$ Department of Information Technology, SVKM's NMIMS, Shirpur, MH, India \\ ${ }^{5}$ Department of Mathematics, National Institute of Technology, Jamshedpur, JH, India \\ ${ }^{6}$ Department of MCA, Jain Deemed to be University, Jayanagar, Bangalore, KA, India
}

\begin{abstract}
The role of a sensor is to sense and gather the information from both remote and domestic zones. Wireless sensor network (WSN) is still emerging to exploit its uses in new extents, though, a lot of works have been done such as habitat monitoring, health care, agriculture, seismic effects, etc. This work focuses on prolonging the lifetime of the WSN through optimizing its energy consumption. The miniature size of the sensor leads to the restrictions with power, lifetime, and memory. In traditional approaches, energy management has been done at the sensor node level whereas, in this work, we focus on cluster-level for energy management. The proposed algorithm named as Residual Energy Adaptive Cluster Head Selection Algorithm (REACH) is a simplified cluster formation process. REACH focuses on uniform energy-based cluster formation for the efficient energy management. The simulation results shows the outperformance of REACH over heterogeneous Stable Election Protocol (SEP) and homogeneous Low Energy Adaptive Clustering Hierarchy $(\mathrm{LEACH})$ in terms of life time and dead node ratio.
\end{abstract}

Key words: Wireless Sensor Network, Heterogeneous, Homogeneous, Residual Energy, Energy Management.

\section{INTRODUCTION}

Wireless Sensor Network is playing a very significant role in collecting information from the environment where human interaction is quite impossible. Many applications have so far developed to maintain the role of sensor nodes, such applications are disaster relief, health care, military defense, etc. The deployment may be centralized or distributed based on network requirements. The implementation of this wireless network was developed by the military to monitor the movement of enemies. Later on, it has immerged to a large extend in various fields as mentioned above. Nowadays nature-inspired meta-heuristic approaches have to make a tremendous change in optimizing SN performance by considering the constraints such as lifetime, energy consumption, memory, etc. Apart from all its limitations, energy optimization is a major concern to prolong the networks lifetime. Initially, every sensor node used to collect the data from the environment and then propagate it to the base station (BS) where a lot of energy is consumed. Later on, the clustering concept brought a breakthrough to reduce the activities of the sensor node to a large extend that consumes less energy as compared to earlier communication strategies. Now, most of the researchers are using clustering techniques as a sub-process to optimize the energy as well as to increase the lifetime of the network. Our work is also motivated by one of the natures inspired approach called the chameleon attack principle [2]. Normally in chameleons attack, the predator approaches towards prey by measuring the gap which must be in the range of its tongue. The sensor nodes in the network are very much limited to its resources and computing capacity, due to its tiny nature [3]. Hence, to increase the lifetime of the sensor network, energy management needs to be focused. Sensor nodes are deployed in various environments where human intervention may be possible or not based on the condition. This is the actual reason why a lot of researches are carried out in the energy management domain of WSN [4]. In this work, we have focused on the above-said constraint.

The implementation of WSN has been extended from domestic to hostile environments like defense, medical, habitat monitoring, natural calamity monitoring etc. The WSN is an indeed technique explicitly for those operations where life risk is there like volcanic or seismic effects. In these situations, battery depletion rate monitoring is one of the primary criteria for accurate monitoring. In this line of thought, the clustering is one of the efficient methods which prolongs the sensor network lifetime [2] [3] [4]. The direct communication between the sensor and base station can be avoided by forming small groups or clusters of sensor nodes. 
Hitesh Mohapatra et al., International Journal of Emerging Trends in Engineering Research, 8(8), August 2020, 4278 - 4286

During this process, several factors can be considered and the inter-neighbor-node (INN) gap and residual energy falls under that [5] [6] [7].

This paper focuses on the improvement of an energy-efficient clustering algorithm by extending the existing algorithms LEACH and SEP. For our work, we have considered homogeneous energy level among sensor nodes and the nature of sensor nodes are static [8]. Our proposed algorithm is a mimic of the chameleon attack which executes in two phases. The first phase implemented the calculation of residual energy of SNs and sorting them in descending order. From the sorted value, the top $10 \%$ of High residual energy-based nodes clubbed in a set called $\mathrm{CH}$-set. And the second part of execution is responsible for cluster formation by measuring the INN gap which is based on nature-inspired phenomena. Our proposed procedure advocates against the adopted sub-operations by our existing traditional algorithms LEACH and SEP. The simulation and evaluation of REACH are compared against the above said traditional algorithms individually. In our clustering process, we also paid the same level of attention to the energy-saving scheme [9] .

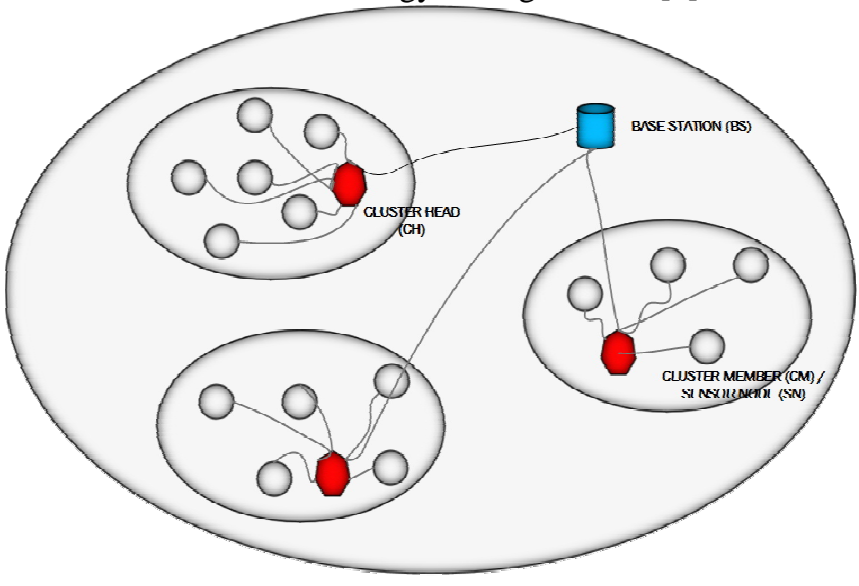

Figure 1: WSN architecture

In a cluster-based design, the SNs are partitioned into a few gatherings known as clusters. Each cluster has an administrative node named as $\mathrm{CH}$. All the SNs sense neighborhood information and pass it to their respective $\mathrm{CH}$ [10]. The CHs at that point total the nearby information lastly send it to the BS straightforwardly or utilizing different CHs. Figure. 1 displays the architecture of a cluster-based WSN. Following are the advantages of a cluster-based WSN:

- The concept of data aggregation is enabled at $\mathrm{CH}$ for eliminating uncorrelated and redundant data. To transmit 1 bit of data, the energy dissipated can also be used in a high amount of data aggregation. Hence the reduction in consumption of energy is done by propagating aggregated data apart from sending a large amount of redundant data [11].
- Since SNs communicate with their CHs, it conserves communication bandwidth and avoids the exchange of redundant pieces of information among themselves [12].

The motivation of this proposed work is to develop an energy-efficient protocol for wireless sensor network which can outperform both in the homogeneous and heterogeneous environment.

The rest of the paper is organized as follows: Section 2 covers literature survey. Section 3 discusses the environment of simulation. Section 4 focuses on proposed REACH algorithm. The result and discussion has been covered under section 5. Section 6 presents the conclusion with future probability.

\section{LITERATURE REVIEW}

The extensive literature survey concludes the variations in the energy management process. Several factors influence the clustering process such as static, dynamic, centralized, or distributed, etc. Out of all such approaches, LEACH (Low Energy Adaptive Clustering Hierarchy) is the hierarchical algorithm that works towards the lifetime of WSN. The random rotation of the cluster head selection method enables the LEACH to maintain the energy balance among the sensor nodes [13]. The LEACH primarily works in a homogeneous environment. The proposed stable election protocol (SEP) is an improvised version of LEACH which works in a heterogeneous environment [14]. This heterogeneous environment is the combination of two types of nodes such as normal node and high energy based node. Called advanced nodes As the advance nodes are used here, the network becomes heterogeneous in terms of the node energy. As the election of $\mathrm{CH}$ is done randomly in LEACH, but here in SEP probability is done by considering the initial energy of each node with the other nodes. It helps in extending the lifetime of the network as the first node dies lately, which is important for some applications $[15,16]$.

In [17] a bio-inspired meta-heuristic cuckoo search was proposed to form clusters in an optimized way. Here the research scholar proposed a nesting concept with high accurate falsie node injection into others nest. In cuckoo search, the fitness function of sensor nodes is calculated by adding weight plus a degree of the node. This approach focuses to extend the life span of the sensor network by reducing the intra-cluster gap. In [18] the integration of harmony and the cuckoo were suggested to enhance the clustering technique and simultaneously an optimized routing protocol. In the initial part of the simulation, a cuckoo search was implemented to detect optimal CHs for clustering, and in the subsequent part; the harmony algorithm helps to choose an optimized route for transferring the information to the BS [19]. The integration of the genetic algorithm and fuzzy 
Hitesh Mohapatra et al., International Journal of Emerging Trends in Engineering Research, 8(8), August 2020, 4278 - 4286

approach proposes a soft-computing based methodology for WSN. This method is the combination of three parameters like density, energy, and centrality [20]. This unique combination of parameters helps to find the most suitable $\mathrm{CH}$ with its position of allocation [21]. Though these algorithms are working fine but it has a major drawback related to cluster formations and cluster membership which leads to a short lifetime for WSN [22].

\section{SYSTEM MODEL}

\subsection{Network Model}

For our simulation, we have considered a bio-inspired meta-heuristic approach. We assume the following constraints such as all SNs are static, the network is the heterogeneous type and $\mathrm{SNs}$ are participating in data exchange. To reduce the amount of energy consumption during data transmission, we used the chameleon attack approach [9]

\subsection{Radio Energy Model}

The proposed algorithm actualizes the comparable technique for the radio model [10-11]. To achieve sensible Signal-to-Noise Ratio (SNR) in exchanging single bit information over a distance $\boldsymbol{d}$, the following equation will be used by us:

$$
\begin{gathered}
E_{T x}(l, d)=l \times E_{\text {elec }}+l \times \varepsilon_{f s} \times d^{2}, \text { if } d<d_{0}(1) \\
E_{T x}(l, d)=l \times E_{\text {elec }}+l \times \varepsilon_{m p} \times d^{4}, \quad \text { if } d \geq d_{0}
\end{gathered}
$$

Here, $\mathbf{E}_{\text {elec }}$ denotes the amount of energy consumed for 1 bit of data transmission, that is used to enable transceiver. The amplification coefficient factors are denoted by $\boldsymbol{\varepsilon}_{\mathrm{fs}}$ and $\boldsymbol{\varepsilon}_{\mathrm{mp}}$. It represents the transmission amplifier in free space and multi-path respectively. $\mathbf{d}_{0}$ symbolizes the threshold value of the transmission distance. And for receiving a single bit of data, the amount of energy dissipated is calculated as follows:

$$
E_{R x}(l)=l \times E_{\text {elec }}(3)
$$

\section{PROPOSED ALGORITHM}

To prolong the network life time, we propose a residual energy-based clustering algorithm, called Residual Energy Adaptive Cluster Head selection algorithm (REACH ) (Ref.Figure 2). The REACH basically considers equal load distribution among the sensor nodes. For that, REACH adopts a sorting mechanism to fom equal energy based clisters. To setup an environment for REACH, few constraints have been assumed or considerd. The constraints are:

1. The WSN is static inature.

2. In WSN all nodes including $\mathrm{CH}$ and BS knows the location of each other..

3. The dominated node $\mathrm{CH}$ is responsible for data accumulation.

4. The considered WSN is homogeneous in nature.

5. Energy investment solely depends upon the data size and distance.

The communication mode within WSN can be segregated into two types such as intra-cluster or inter-cluster communication mode [23]. The clustering process is dependent upon inter-cluster communication mode. The data transmission between sensor ndoes and CHs can made either through single-hop or multi-hop communication mode [24]. The $\mathrm{CH}$ is responsible to integrate the collcted data from indivudial sensors by removing unnecessary data. Further, the $\mathrm{CH}$ forwards that integrated data to the BS.

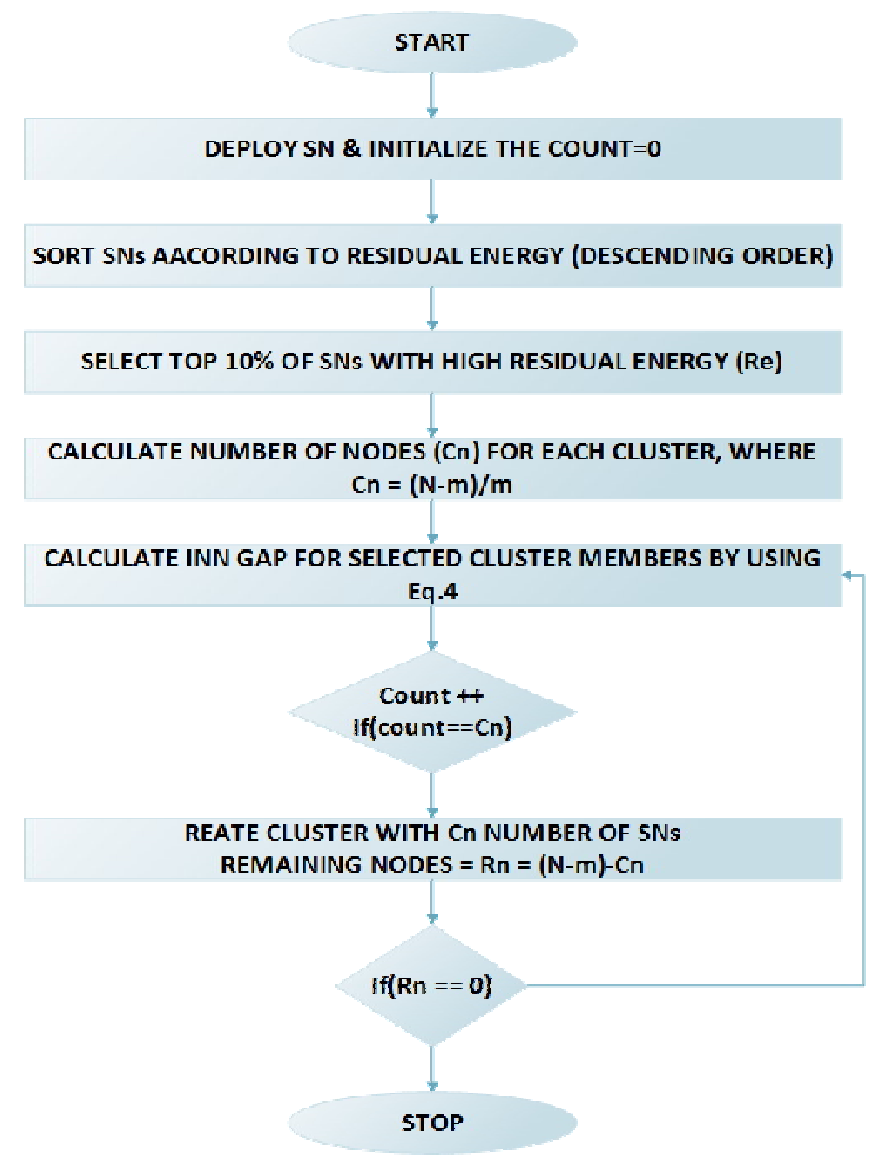

Figure 2: Flowchart of Proposed Scheme (REACH)

This indirect communication puts less pressure on the sensor nodes which leads to a long lifetime for the SNs and the network. By selecting a specific node as $\mathrm{CH}$ for each round leads to rapid energy depletion and that leads to new $\mathrm{CH}$ selection rounds. The cluster head selection round itself consumes a substantial amount of energy. So, by reducing such $\mathrm{CH}$ selection round the network life span can be enhanced. The proposed REACH algorithm works with the above theme where it creates equal energy-based clusters and 
Hitesh Mohapatra et al., International Journal of Emerging Trends in Engineering Research, 8(8), August 2020, 4278 - 4286

reduces frequent $\mathrm{CH}$ selection processes. The residual energy $\left(\mathbf{R}_{\mathbf{e}}\right)$ is the criteria for selecting the new $\mathrm{CH}$.

\section{Algorithm: REACH Algorithm (Proposed) \\ Assumption: Sensor nodes (SNs) are homogeneous by considering identical residual energy $\left(\mathrm{R}_{\mathrm{e}}\right)$. Where, $R_{e}=$ Residual Energy, $N=$ Number of Sensor Nodes, $m=$ Number of Cluster Heads, $C_{n}=$ Number of SNs in each Cluster, $\mathrm{CH}=$ Cluster Head).}

Input: Set of $\mathrm{SNs} S=\left\{\mathrm{S}_{1}, \mathrm{~S}_{2} \ldots \mathrm{S}_{\mathrm{N}}\right\}$ and $\mathrm{N}$ is the total number of $\mathrm{SNs}$. $\mathrm{R}_{\mathrm{e}}$ of individual nodes is provided.

Output: Optimized cluster formation and optimized lifetime of the cluster.

\section{Step 1: Initialization}

For $\mathrm{i}=1$ to $\mathrm{N}$

Evaluate the $\mathrm{R}_{\mathrm{e}}$ of each $\mathrm{SN}$.

End

Sort, SNs according to $\mathrm{R}_{\mathrm{e}}$ in descending order.

Step 2: Calculate the number of SNs in a particular cluster using Eq. 4

$\mathrm{C}_{\mathrm{n}}=(\mathrm{N}-\mathrm{m}) / \mathrm{m} \ldots(4)$

Form CH-set with top 'm' SNs from the above-sorted list.

Step 3: Evaluate the INN gap of $\mathrm{CH}$-set from the SNs using Eq. 5

For each node $n_{i}, i=1,2, \ldots,(N-m)$

Calculate distance $\mathrm{d}\left(\mathrm{n}_{\mathrm{i}}, \mathrm{CH}_{\mathrm{e}}\right)$ between $\mathrm{CHs}$ and node $\mathrm{n}_{\mathrm{i}}$,

$$
d\left(n_{i}, C H \theta\right)=\text { min }_{4 g=1,2, m_{m}} d\left(n_{*}, C H \theta\right), \ldots(5)
$$

Step 4: Restrict cluster population

Initialize count $=0$

If $\left(\right.$ count $==\mathrm{C}_{\mathrm{n}}$ )

Then (Form cluster)

Do \{

(Remaining Node) $\mathrm{R}_{\mathrm{mn}}=(\mathrm{N}-\mathrm{m})-\mathrm{C}_{\mathrm{n}} \ldots$ (6)

\} while $\left(\mathrm{C}_{\mathrm{n}}==0\right)$

Repeat Step 4 for each $\mathrm{m}$ while condition is true

Step 5: Repeat Steps (1-4) for new $\mathrm{CH}$ selection.

Step 6: Stop

A clustering-based protocol consists of four stages such as; (i)

$\mathrm{CH}$ selection stage, (ii) Cluster formation stage,(iii) data aggregation stage, and (iv) data communication stage [25].

This whole process executes through two phases that are, setup phase and steady-state phase [26,27]. During the set-up phase, the sensor nodes are intimating about their remaining energy level with location information to the BS. Based on this data the BS calculated the average energy consumption by the sensor network. As $\mathrm{CH}$ is responsible for many other works like data aggregation, forwarding data to the BS hence, the sensor nodes which has the highest $\mathbf{R}_{\mathbf{e}}$ is elected as $\mathrm{CH}$. The proposed REACH algorithm makes ensure that the node with the highest $\mathbf{R}_{\mathbf{e}}$ is only elected as $\mathrm{CH}[28,29]$.

The execution of the REACH algorithm has been processed through the following phases: In the initialization phase, SNs are randomly deployed in a target area. Next, the SNs are sorted according to $\mathbf{R}_{\mathbf{e}}$ in descending order. Let the sorted list is represented as $\mathrm{S}$. In the $\mathrm{CH}$ selection phase, to select the best $\mathrm{CH}$, top $10 \% \mathrm{SNs}$ have been chosen from the list $\mathrm{S}$ and a $\mathrm{CH}$-set is formed. Now, these selected nodes will behave as a $\mathrm{CH}$. In the third phase, the INN (Inter Neighbor Node) gap is evaluated, which is nothing but selecting cluster members for the CHs. The selection of cluster member processes for $\mathrm{CH}$ is inspired by nature i.e. the way chameleon attacks its prey. If the prey is coming under the range of chameleon's tongue length then it attacks that prey. In the same way, $\mathrm{CH}$ selects its member by evaluating the INN gap. Cluster formation phase: This is the final step of the setup phase.

REACH has adopted a different mechanism of cluster formation. Initially, one cluster member is assigned to each $\mathrm{CH}$ by using the INN gap. Suppose $\mathrm{CH} 1$ founds its first cluster member then $\mathrm{CH} 2$ will find its cluster member and this will continue till the last $\mathrm{CHn}$. Again, the same procedure will continue for the next cluster member from $\mathrm{CH} 1$ to $\mathrm{CHn}$. This will help in the uniform distribution of SNs in respective clusters. After cluster formation, data aggregation and data communication phases take place. The proposed REACH is unique in terms of several factors such as:

- It avoids the frequent involvement of SNs in the cluster formation process.

- It prolongs the network lifetime by voiding non-uniform cluster formation by considering the energy level.

- It maintains a synchronous energy depletion policy so that the entire cluster will uniformly deplete energy.

- The proposed algorithm has been compared against both homogeneous and heterogeneous clustering protocols.

The limitation of the proposed clustering scheme is, when the density of SNs increases it leads to form more numbers of clusters and CHs. This enhances the complexity of the algorithm. Secondly, the distribution of SNs must be scattered rather than clubbed. During clubbed environment, it may all the $\mathrm{CHs}$ or maximum of $\mathrm{CHs}$ could be deployed in a specific portion of the target zone. It happens as per the algorithm if the INN gap will be more between SNs and $\mathrm{CH}$ and that leads to more energy investment processes.

\section{SIMULATION AND RESULTS}

Table 1: Network Parameters

\begin{tabular}{|l|l|}
\hline Parameters & Value \\
\hline Number of nodes & 100 \\
\hline BS Location & $(50,50)$ \\
\hline Network Size & $100 \times 100$ \\
\hline$E_{\text {elec }}$ & $50 \mathrm{~nJ} / \mathrm{bit}$ \\
\hline$E_{m p}$ & $0.0013 \mathrm{pJ} / \mathrm{bit} / \mathrm{m}^{4}$ \\
\hline$E_{f s}$ & $100 \mathrm{pJ} / \mathrm{bit} / \mathrm{m}^{2}$ \\
\hline$E_{\text {aggregate }}$ & $5 \mathrm{n} \mathrm{J} / \mathrm{bit} / \mathrm{signal}^{4}$ \\
\hline Percentage of CHs & $10 \%$ of the total nodes \\
\hline Initial Energy & $0.5 \mathrm{~J} / \mathrm{node}$ \\
\hline Packet size & $4000 \mathrm{bits}$ \\
\hline
\end{tabular}


Hitesh Mohapatra et al., International Journal of Emerging Trends in Engineering Research, 8(8), August 2020, 4278 - 4286

The supercomputer (PARAM SHAVAK) with MATLAB-R2016A is used for the execution of the REACH algorithm [27]. An area of $(100 \mathrm{~m} \times 100 \mathrm{~m})$ with 100 nodes is used as the setup environment. The initial energy for all the sensor nodes SNs is equal i.e. 0.5 Joule for each node. To maintain an approximately equal distance between the $\mathrm{CHs}$ and the BS the $\mathrm{CH}$ has been deployed at the location $(50,50)$ that represents the center point of the network. The other considered parameters are mentioned in Table 1 . The second step of REACH includes sorting of SNs according to the Re whereas, it is not true for the first iteration due to the homogeneous nature of the network. From the 2 nd round till the end of the process, the homogeneous network converted to heterogeneous type by varying Re. Hence, we have compared our bio-inspired meta-heuristic algorithm with both homogeneous and heterogeneous existing protocols such as LEACH and SEP respectively. The simulation of REACH is carried out in two scenarios.

\subsection{Scenario 1: Homogeneous Environment}

Initially, all the SNs will have 0.5 Joule as startup energy. Around $10 \%$ of the total nodes will be elected as CHs. The CHs will form clusters by applying "(4)".

$$
d\left(n_{i}, C H e\right)=m i n_{\psi \varepsilon=1}, 2_{m,} m\left\{d\left(n_{i}, C H e\right)\right\} \text { (4) }
$$

Where, $\boldsymbol{n}$ denotes the number of nodes, $\mathrm{CH}$ as cluster head. The 2nd part of the equation calculates the minimum distance between $\boldsymbol{n}$ and ' $\mathrm{CH}$ ' by varying $\mathrm{i}=1,2,3 \ldots \mathrm{n}$. Like other traditional homogeneous network protocols. We have done the comparison with the existing LEACH [22] protocol which is homogeneous. Figures. 3(a) and 3(c), presents the ratio between the number of dead nodes with the number of rounds for both REACH and LEACH respectively. Figures.3(b) and 3(d) presents the comparison between REACH and LEACH in terms of total average energy consumption respectively. The numerical computation of results represented in Table 2 . In Figures.3 (a,b,c,d), we have presented the ratio between the number of dead nodes against numbers of rounds for both REACH and LEACH respectively.

Table.2: Comparison between REACH and LEACH

\begin{tabular}{|c|c|c|}
\hline $\begin{array}{c}\text { PROTOCOL } \\
\text { S }\end{array}$ & LEACH & REACH (Proposed) \\
\hline FND & 509 & $\mathbf{1 3 2 0}$ \\
\hline HNA & 792 & $\mathbf{1 5 5 7}$ \\
\hline AND & 1174 & $\mathbf{1 6 7 4}$ \\
\hline
\end{tabular}

Table 2 illustrates the performance of REACH over LEACH. The performance has been recorded at different levels of (three different instances) such as First Node Dead (FND), Half Node Alive (HNA), and All Node Dead (AND). From this simulation REACH performs in an optimized way in comparison with LEACH by $41 \%, 39 \%$, and $25 \%$ during FND, HNA, and AND respectively.

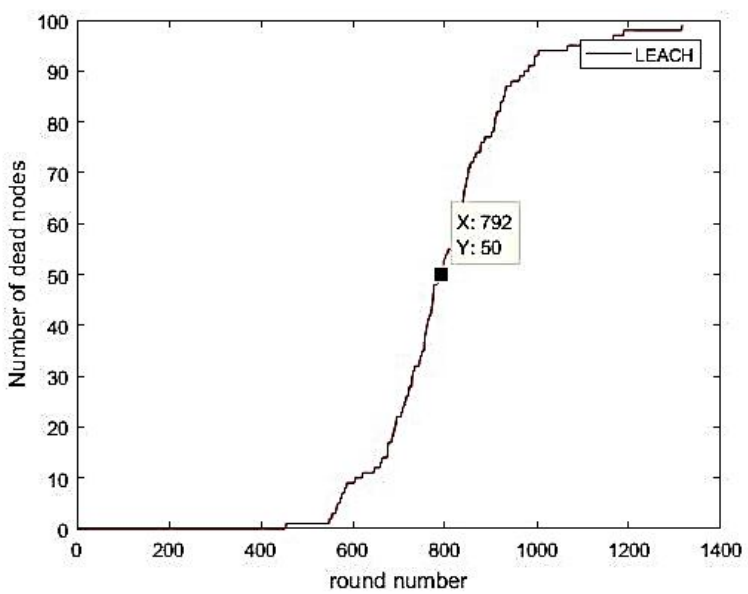

Figure 3: (a) 50\% of nodes are alive in LEACH.

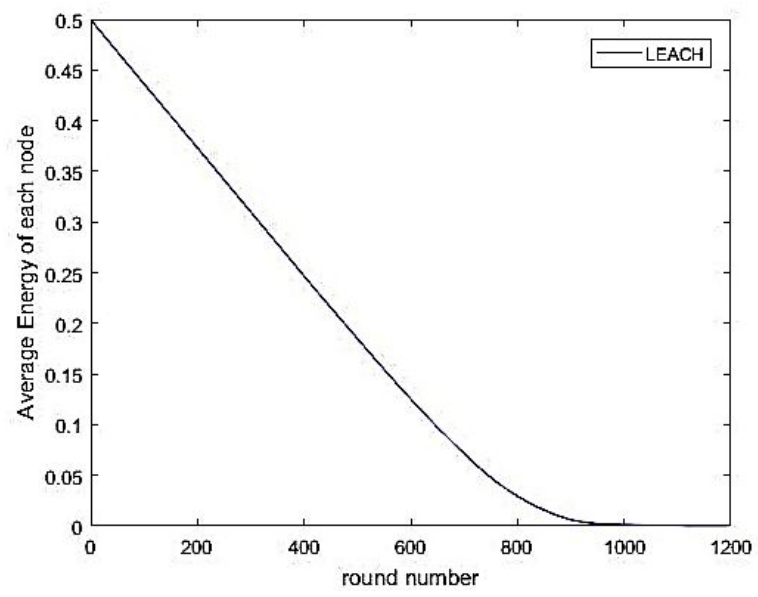

Figure 3: (b) Energy consumed by LEACH protocol.

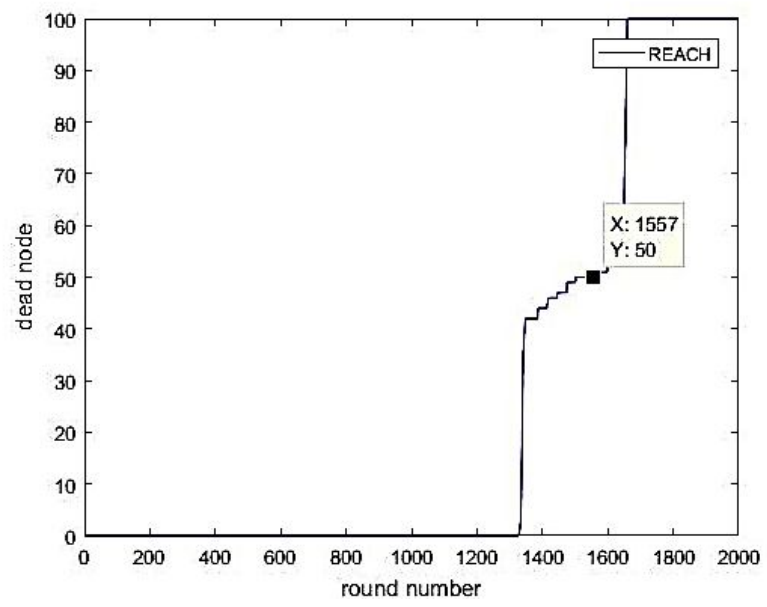

Figure 3: (c) 50\% of nodes are alive in REACH. 


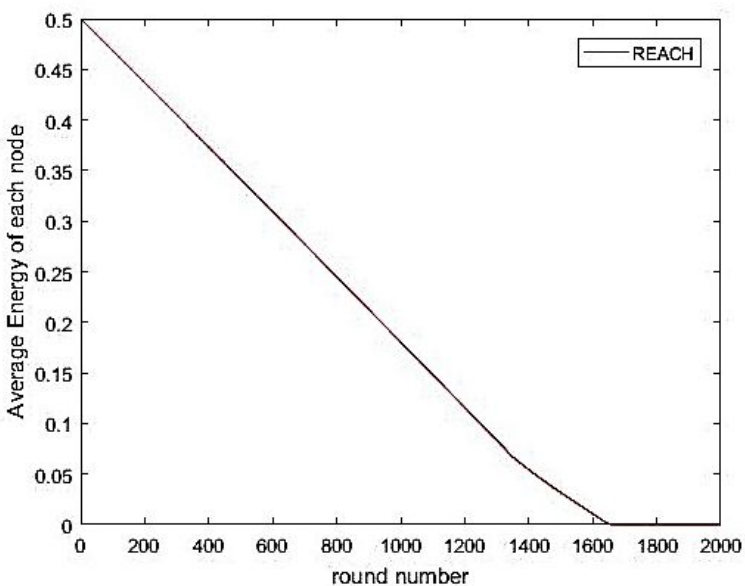

Figure 3: (d) Energy consumed by REACH protocol.

\subsection{Scenario 2: Heterogeneous Environment}

After the first iteration, the considered homogeneous network converts into a heterogeneous sensor network. After the first electoral round, there is a variation in the Re level of the SNs. According to the REACH algorithm, we have sorted the SNs according to their Re level in descending order. The top 10\% of the sorted SNs have elected as $\mathrm{CH}$ for that round. In the second step of REACH, the remaining SNs excluding CHs need to be clubbed with $\mathrm{CH}$ to form clusters. In the proposed REACH model, we have used the INN gap distance to allocate SNs as a cluster member for a particular $\mathrm{CH}$ during each round. Initially, the $\mathrm{CH} 1$ will choose the nearby $\mathrm{SN}$ as its cluster member. Likewise, the rest of the $\mathrm{CHs}$ will choose their cluster member.

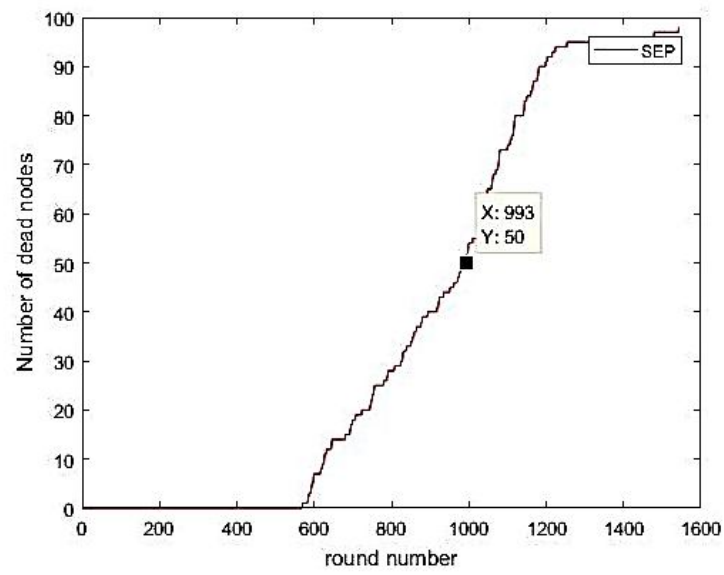

Figure 4: (a) 50\% of nodes are alive in SEP.

This cluster-member assigning process will continue until the last node of the sensor network. After cluster formation like other traditional WSN protocols, the data aggregation, and segregation process will continue. After completion of data transmission of aggregated data from $\mathrm{CHs}$ to $\mathrm{BS}$ is over. Again, all the SNs including CHs will be sorted and the above said process will iterate. We have done the comparison with the existing SEP protocol which is heterogeneous. Figures. $(\mathrm{a}, \mathrm{b}, \mathrm{c}, \mathrm{d})$ represents the comparison between REACH and SEP in terms of total average energy consumption respectively. The numerical computation of results represented in Table 3.

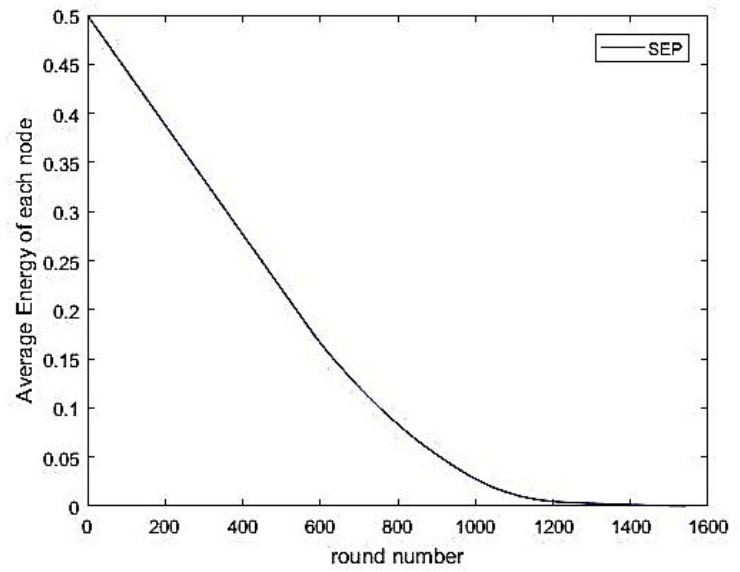

Figure 4: (b) Energy consumed by SEP protocol.

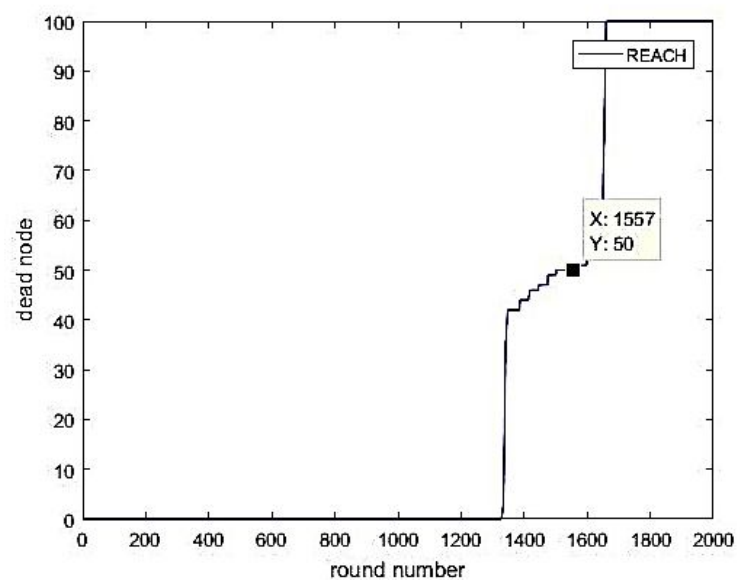

Figure 4: (c) 50\% of nodes are alive in REACH.

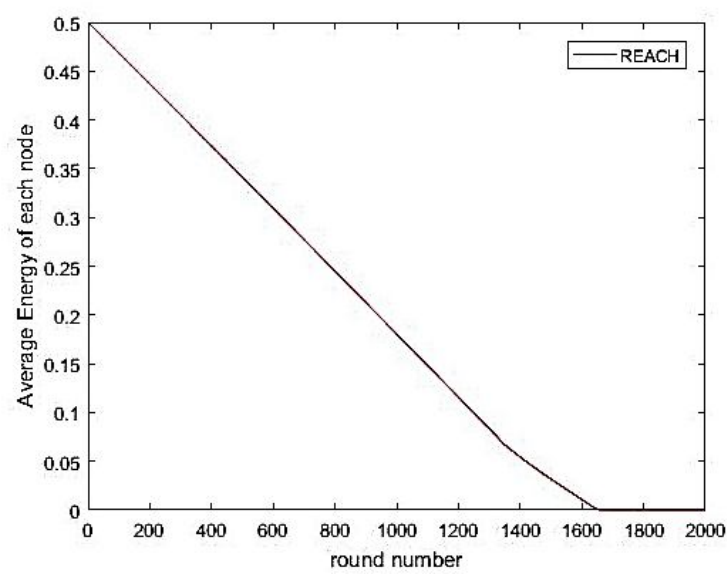

Figure 4: (d) Energy consumed by REACH protocol.

Table 3 presents the performance comparison between REACH and SEP. The performances has been recorded at 3 
Hitesh Mohapatra et al., International Journal of Emerging Trends in Engineering Research, 8(8), August 2020, 4278 - 4286

distinct positions such as first node dead (FND), half node alive (HNA), and all node dead (AND). From this simulation REACH performs in an optimized way in comparison with SEP by $39 \%, 31 \%$, and $11 \%$ during FND, HNA, and AND respectively. The performance comparison of proposed REACH against LEACH and SEP proves the outperformance both in a homogeneous and heterogeneous environment. The cumulative comparison graph claims the better performance of REACH.

Table 3: Comparison between SEP and LEACH.

\begin{tabular}{|c|c|c|}
\hline $\begin{array}{c}\text { PROTOCOL } \\
\text { S }\end{array}$ & SEP & REACH (Proposed) \\
\hline FND & 539 & $\mathbf{1 3 2 0}$ \\
\hline HNA & 993 & $\mathbf{1 5 5 7}$ \\
\hline AND & 1456 & $\mathbf{1 6 7 4}$ \\
\hline
\end{tabular}

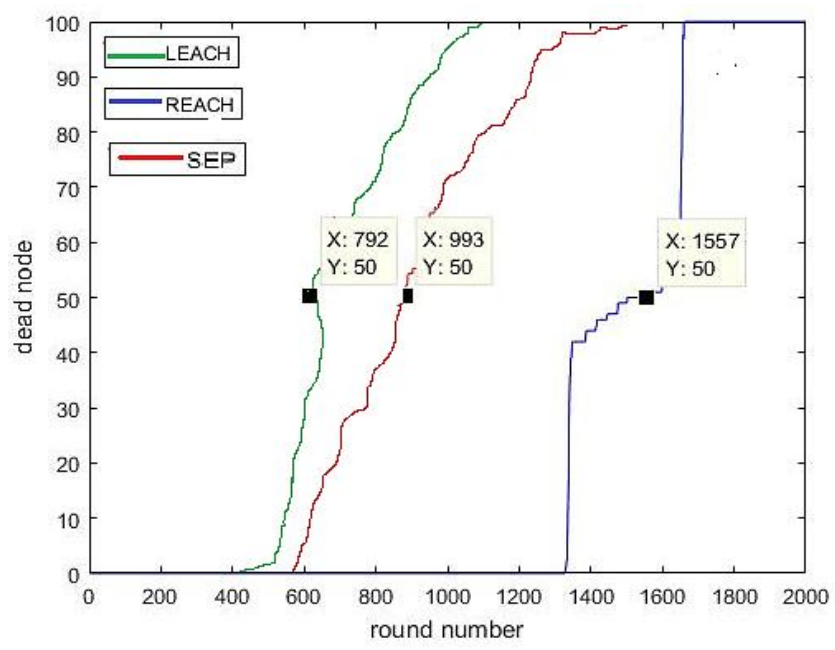

Figure 5: Comparison of Network Lifetime after 50\% of nodes are alive in REACH, SEP, and LEACH.

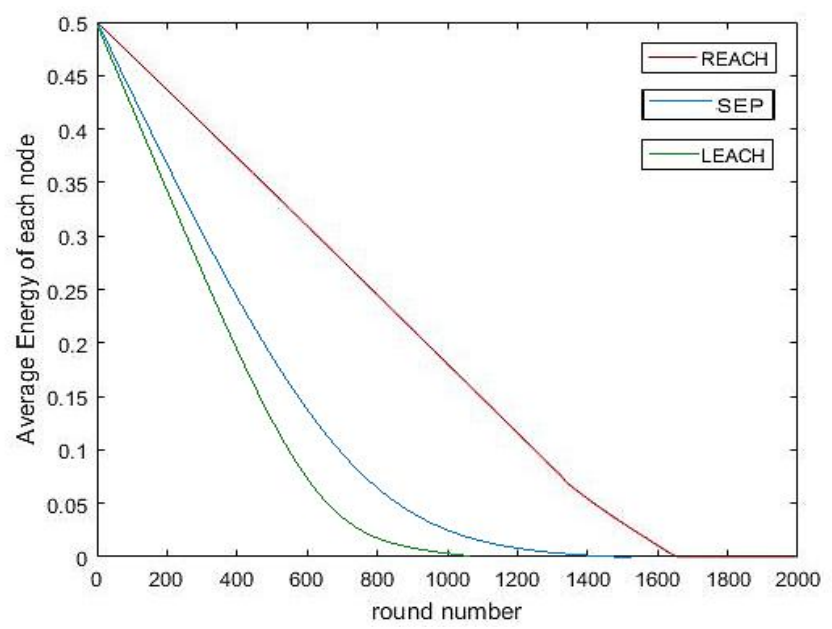

Figure 6: Comparison of Average Energy consumed by REACH, SEP, and LEACH protocol.
The character comparison among LEACH, SEP, and REACH is illustrated in Table. 4 (Ref. Figures 5 and 6). For this comparison, we have considered a few explicit properties of these said protocols. The properties are: heterogeneity level, clustering method, location information, cluster information, sensitivity, intra-cluster communication, $\mathrm{CH}$ and $\mathrm{BS}$ communication mode, probability, neighborhood information, and presence of faults. These are basic character for any sensor network and considering those properties of the REACH protocol has been explored by exploiting LEACH and SEP protocols.

\subsection{Complexity Analysis}

This sub-section discusses the complexity analysis of these stated algorithms. During LEACH, the $\mathrm{CH}$ selection process is purely based on the threshold value of the SNs. Every SN of the cluster maintenance a different level of threshold which concludes that the complexity of LEACH is: $\mathrm{O}(\mathrm{n})$. In the case of SEP, it uses two types of nodes like normal SN and advanced SN. The probability of getting $\mathrm{CH}$ is based on the threshold values of two types of nodes hence; the complexity of SEP is $\mathrm{O}(2 n)$. The proposed REACH protocol is dividing the whole SNs set based on the sorted residual energy level. If there is an $m$ number of clusters that need to form from $n$ numbers of SNs, in that case, the complexity will be $\mathrm{O}(\mathrm{n} / \mathrm{m})$. The present complexity analysis proves the efficiency of the proposed REACH algorithm (Ref: Table 4).

Table 4: Comparitative anlysis

\begin{tabular}{|l|l|l|l|}
\hline REACH & SEP & LEACH & Protocols \\
\hline Yes/2 & Yes/2 & No/- & Heterogeneity and Level \\
\hline D & D & D & Clustering Method(Centralize(C)/Decentralized(D) Mixed(M) \\
\hline N & N & N & Location Information (Y/N) \\
\hline F/V/V & F/V/V & V/V/V & Cluster (Count/Size/Density) Variable (V)/ Fixed (F) \\
\hline Y & Y & Y & Existence of Sensitivity (YN) \\
\hline Multi-Hop & 1-Hop & 1-Hop & Intra-cluster Communication \\
\hline D/ & I & D & CH and BS Communication Direct (D)/Indirect (I) \\
\hline W & W & W & Probability (Pure(P)/Weighted(W)) \\
\hline Y/Y & Y/Y & Y/Y & Neighborhood Distance/Location)(Y/N) \\
\hline C & C & C & Fault- Clustering(C)Routing:(R)) \\
\hline
\end{tabular}

\section{CONCLUSION}

In this paper, an advanced hierarchical clustering algorithm named REACH has been discussed with proper validation against exiting clustering algorithms LEACH and SEP. The uniqueness of the validation is the proposed REACH has been evaluated against both homogeneous and heterogeneous algorithms. From the simulation it has been observed that the reach outperforms by $37 \%$ and $27 \%$ over LEACH and SEP. Enegy management is a trending domain of research. The state-of-the-art can be enhanced by considering dynamic sensor deployment scenario. In real-time, the REACH is more suitable for unattainable SNs deployment structures like aquatic monitoring, volcanic monitoring, etc. This work 
Hitesh Mohapatra et al., International Journal of Emerging Trends in Engineering Research, 8(8), August 2020,4278 - 4286

gives an insight towards the equal distribution and reducing the frequent $\mathrm{CH}$ selection process. To motivate the readers, few relevant research under uncertainty [30-38] have been suggested.

\section{REFERENCES}

[1] MA. Adnan, MA. Razzaque, A. Abedin, SM. Salim Reza, and MR. Hussein, A novel cuckoo search based clustering algorithm for wireless sensor networks, Advanced Computer and Communication Engineering Technology, Springer, pp. 621-634, 2016.

[2] H. Mohapatra and AK. Rath, Fault tolerance in wsn through pe-leach protocol, IET Wireless Sensor Systems, vol. 9, no. 6, pp. 358-365, 2019

[3] G. Smaragdakis, I Matta, and A Bestavros, SEP: a stable election protocol for clustered heterogeneous wireless sensor networks, pp. 1-11, 2004.

[4] S. Lindsey and CS. Raghavendra, PEGASIS: Power-efficient gathering in sensor information systems, in Proceedings, IEEE Aerospace Conference, Big Sky, MT, USA, pp. 1-3, 2002.

[5] H. Mohapatra and A.K Rath, Fault tolerance through energy balanced cluster formation (EBCF) in WSN, in Smart Innovations in Communication and Computational Sciences. Advances in Intelligent Systems and Computing, Singapore, vol. 851, pp. 313-321, 2019, https://doi.org/ 10.1007/978-981-13-2414-7_29.

[6] WR. Heinzelman, A. Chandrakasan, and H. Balakrishnan, Energy-efficient communication protocol for wireless microsensor networks, Proceedings of the 33rd Annual Hawaii International Conference on System Sciences, USA, 2000. doi: 10.1109/HICSS.2000.926982

[7] WB. Heinzelman, AP. Chandrakasan, and $H$. Balakrishnan, An application-specific protocol architecture for wireless microsensor networks, IEEE Transactions on Wireless Communications, vol. 1, no. 4, pp. 660-670, 2002. https://doi.org/10.1109/TWC.2002.804190

[8] G. Karypis, EH. Han, and V. Kumar, Chameleon: hierarchical clustering using dynamic modeling, IEEE Computer, vol. 32, pp. 68-75, 1999.

[9] H. Mohapatra and AK. Rath, Survey on fault tolerance-based clustering evolution in WSN, IET Networks, vol. 9, no. 4, pp. 145-155, 2020, doi: 10.1049/iet-net.2019.0155.

[10] C. Buratti, A. Conti, D. Dardari, and R. Verdone, An overview on wireless sensor networks technology and evolution, Sensors, vol. 9, no. 9, pp 6869-6896, 2009.
[11] H. Çam, S. Özdemir, P. Nair, D. Muthuavinashiappan, and HO. Sanli, Energy-efficient secure pattern-based data aggregation for wireless sensor networks, Computer Communications, vol. 29, no. 4, pp. 446-455, 2006.

[12] AA. Abbasi and M. Younis, A survey on clustering algorithms for wireless sensor networks, Comput. Commun, vol. 30, no. 14-15, pp. 2826-2841, 2007.

[13] MS. BenSaleh, R. Saida, YH. Kacem, and M. Abid, Wireless sensor network design methodologies: a survey, Journal of Sensors, pp. 1-13, 2020 https://doi.org/10.1155/2020/9592836

[14] FA Aoudia, M. Gautier and O. Berder, Distributed computation of fair packet rates in energy harvesting wireless sensor networks, IEEE Wireless Communications Letters, vol. 6, no. 5, pp. 626-629, 2017.

[15] I. Krikidis, Average age of information in wireless powered sensor networks, IEEE Wireless Communications Letters, vol. 8, no. 2, pp. 628-631, 2019.

[16] H. Mohapatra and AK. Rath, IoT-based smart water, in IoT Technologies in Smart Cities: From sensors to big data, security and trust. UK: IET, pp. 63-82, 2020

[17] TN. Le, A. Pegatoquet, O. Berder, and O. Sentieys, Energy-efficient power manager and mac protocol for multi-hop wireless sensor networks powered by periodic energy harvesting sources, IEEE Sensors Journal, vol. 15, no. 12, pp. 7208-7220, 2015

[18] PV. Mekikis, E. Kartsakli, A. Antonopoulos, L. Alonso, and C. Verikoukis, Connectivity analysis in clustered wireless sensor networks powered by solar energy, IEEE Transactions on Wireless Communications, vol. 17, no. 4, pp. 2389-2401, 2018.

[19] H. Mohapatra and AK. Rath, Detection and avoidance of water loss through municipality taps in India by using smart taps and ICT, IET Wireless Sensor Systems, vol. 9, no. 6, pp. 447-457, 2019.

[20] M. Panda, P. Pradhan, H. Mohapatra, NK Barpanda, Fault tolerant routing in heterogeneous environment, International Journal of Scientific \& Technology Research, vol. 8, no. 8, pp. 1009-1013, 2019.

[21] H. Mohapatra and AK. Rath, Fault tolerance in wsn through uniform load distribution function, International Journal of Sensors, Wireless Communications and Control, vol. 10, no. 1, pp. 1-10, 2020.

[22] H. Panda, H. Mohapatra, and AK. Rath, "WSN-based water channelization: an approach 
of smart water," in Smart Cities-Opportunities and Challenges. Lecture Notes in Civil Engineering, Singapore, vol. 58, 2020.

[23] CDAC, "PARAM Shavak - Supercomputing Solution in a Box," Pune, 2016.

[24] M. Shopon, MA. Adnan, and MF. Mridha, Krill herd based clustering algorithm for wireless sensor networks, in International Workshop on Computational Intelligence (IWCI), Dhaka, pp. 96-100, 2016.

[25] GG. Wang, AH. Gandomi, XS. Yang, and AH. Alavi, A new hybrid method based on krill herd and cuckoo search for global optimisation tasks, Int. J. Bio-Inspired Comput., vol. 8, no. 5, pp. 286-299, 2016.

[26] H. Mohapatra and AK. Rath, Fault-tolerant mechanism for wireless sensor network, IET Wireless Sensor Systems, vol. 10, no. 1, pp. 23-30, 2020.

https://doi.org/10.1049/iet-wss.2019.0106

[27] H. Mohapatra, S. Rath, S. Panda, R. Kumar, Handling of man-in-the-middle attack in wsn through intrusion detection system, International Journal of Emerging Trends in Engineering Research, vol. 8, no. 5, pp. 1503-1510, 2020.

[28] H. Mohapatra, S. Panda, AK. Rath, SA Edalatpanah, R. Kumar, A tutorial on powershell pipeline and its loopholes, International Journal of Emerging Trends in Engineering Research, vol. 8, no. 4, pp-975-982, 2020.

[29] SA. Mohammed, K. abd Elsalam Aly, AM. Ghuniem, An enhancement process for reducing energy consumption in wireless sensor network, International Journal of Emerging Trends in Engineering Research, vol. 8, no. 6, pp. 2765-2769, 2020.

[30] R. Kumar, SA Edalatpanah, H Mohapatra, A note on "Optimal path selection approach for fuzzy reliable shortest path problem. Journal of Intelligent \& Fuzzy Systems, pp. 1-4, 2020

[31] S. Gayen, S. Jha, M. Singh and R. Kumar, On a generalized notion of anti-fuzzy subgroup and some characterizations, International Journal of Engineering and Advanced Technology, vol. 8, pp. 385-390, 2019.

[32] R. Kumar, SA. Edalatpanah, S. Jha, S. Gayen and R. Singh, shortest path problems using fuzzy weighted arc length, International Journal of Innovative Technology and Exploring Engineering, vol. 8, pp. 724-731, 2019.

[33] S. Gayen, F. Smarandache, S. Jha, M. K. Singh, S. Broumi and R. Kumar, Introduction to plithogenic hypersoft subgroup, Neutrosophic Sets and Systems, vol. 33, pp. 208-233, 2020.
[34] . R. Kumar, S. Jha and R. Singh, A different approach for solving the shortest path problem under mixed fuzzy environment, International Journal of Fuzzy System Applications, vol. 9, no. 2, pp. 132-161, 2020.

[35] R. Kumar, SA. Edalatpanah, S. Jha, S. Broumi, R. Singh and A. Dey, A multi objective programming approach to solve integer valued neutrosophic shortest path problems, Neutrosophic Sets and Systems, vol. 24, pp. 134-149, 2019.

[36] R Kumar, SA Edalatpanah, S. Jha, and R Singh, A novel approach to solve gaussian valued neutrosophic shortest path problems, International Journal of Engineering and Advanced Technology, vol. 8, no. 3, pp. 347-353, 2019

[37] R Kumar, SA Edalatpanah, S. Jha, and R. Singh, A Pythagorean fuzzy approach to the transportation problem, Complex \& Intelligent Systems, vol. 5, no. 2, pp. 255-263, 2019. https://doi.org/10.1007/s40747-019-0108-1

[38] R Kumar, SA Edalatpanah, S. Jha, S. Broumi, and A. Dey, Neutrosophic shortest path problems, Neutrosophic Sets and Systems, vol. 23, pp. 5-15, 2018. 